ARTICLE

\title{
In-situ structure and catalytic mechanism of NiFe and CoFe layered double hydroxides during oxygen evolution
}

Fabio Dionigi ${ }^{1,9 凶}$, Zhenhua Zeng (10) ${ }^{2,9 凶}$, Ilya Sinev ${ }^{3,4}$, Thomas Merzdorf ${ }^{1}$, Siddharth Deshpande ${ }^{2}$, Miguel Bernal Lopez ${ }^{3,4}$, Sebastian Kunze ${ }^{3,4}$, loannis Zegkinoglou (10 3,4, Hannes Sarodnik', Dingxin Fan², Arno Bergmann ${ }^{1,4}$, Jakub Drnec (10 5 ${ }^{5}$, Jorge Ferreira de Araujo ${ }^{1}$, Manuel Gliech', Detre Teschner 6,7 , Jing Zhu ${ }^{8}$, Wei-Xue Li (D) ${ }^{8}$, Jeffrey Greeley ${ }^{2}$, Beatriz Roldan Cuenya (i) ${ }^{4 凶} \&$ Peter Strasser (iD ${ }^{1 凶}$

NiFe and CoFe (MFe) layered double hydroxides (LDHs) are among the most active electrocatalysts for the alkaline oxygen evolution reaction (OER). Herein, we combine electrochemical measurements, operando X-ray scattering and absorption spectroscopy, and density functional theory (DFT) calculations to elucidate the catalytically active phase, reaction center and the OER mechanism. We provide the first direct atomic-scale evidence that, under applied anodic potentials, MFe LDHs oxidize from as-prepared $\alpha$-phases to activated $\gamma$ phases. The OER-active $\gamma$-phases are characterized by about $8 \%$ contraction of the lattice spacing and switching of the intercalated ions. DFT calculations reveal that the OER proceeds via a Mars van Krevelen mechanism. The flexible electronic structure of the surface Fe sites, and their synergy with nearest-neighbor $M$ sites through formation of O-bridged Fe-M reaction centers, stabilize OER intermediates that are unfavorable on pure $M-M$ centers and single Fe sites, fundamentally accounting for the high catalytic activity of MFe LDHs.

\footnotetext{
${ }^{1}$ The Electrochemical Energy, Catalysis, and Materials Science Laboratory, Department of Chemistry, Chemical Engineering Division, Technical University Berlin, Strasse des 17. Juni 124, 10623 Berlin, Germany. ${ }^{2}$ Davidson School of Chemical Engineering, Purdue University, West Lafayette, Indiana 47907, USA. ${ }^{3}$ Department of Physics, Ruhr-University Bochum, Universitaetsstrasse 150, 44801 Bochum, Germany. ${ }^{4}$ Department of Interface Science, Fritz-HaberInstitut der Max-Planck-Gesellschaft, Faradayweg 4 - 6, 14195 Berlin, Germany. ${ }^{5}$ European Synchrotron Radiation Facility, ID 31 Beamline, BP 220 , F-38043 Grenoble, France. ${ }^{6}$ Department of Inorganic Chemistry, Fritz-Haber-Institut der Max-Planck-Gesellschaft, Faradayweg 4-6, 14195 Berlin, Germany. ${ }^{7}$ Max Planck Institute for Chemical Energy Conversion, Stiftstrasse 34-36, 45470 Mülheim an der Ruhr, Germany. ${ }^{8}$ CAS Excellence Center for Nanoscience, Hefei National Laboratory for Physical Sciences at Microscale, School of Chemistry and Materials Science, University of Science and Technology of China, Hefei, Anhui 230026, China. ${ }^{9}$ These authors contributed equally: Fabio Dionigi, Zhenhua Zeng. ${ }^{\circledR}$ email: fabio.dionigi@tu-berlin.de; zeng46@purdue.edu; roldan@fhi-berlin.mpg.de; pstrasser@tu-berlin.de
} 
W ater splitting to generate $\mathrm{O}_{2}$ and $\mathrm{H}_{2}$ has been a major focus of (photo)electrochemical energy storage and conversion research, but fundamental and practical challenges remain. In this process, $\mathrm{O}_{2}$ generation at the anode through the oxygen evolution reaction (OER), which is inherently slower by over four orders of magnitude compared with $\mathrm{H}_{2}$ generation, accounts for the majority of energy losses ${ }^{1}$. NiFebased layered hydroxides are the most active OER catalysts in base and are the catalysts of choice for industrial water electrolysis ${ }^{2-10}$, whereas CoFe-based layered hydroxides have comparable performance $7,8,10-12$. Very recently, it has been found that $\mathrm{NiFe}$ and $\mathrm{CoFe}(\mathrm{MFe})$ layered (oxy)hydroxides are also the common active phases of other highly active OER catalysts, including perovskite oxides ${ }^{13,14}$, spinel oxides ${ }^{15}$, phosphides $^{16}$, and potentially other $\mathrm{Co}-$ and Ni-based OER catalysts with $\mathrm{Fe}$ incorporated intentionally or accidentally, such as carbides ${ }^{17}$, nitrides $^{18}$, sulfides ${ }^{19}$, and selenides ${ }^{20}$, which are prone to hydrolysis and oxidation under OER conditions ${ }^{13,15,16,21,22}$. Thus, studying the reactive structures of the MFe layered double hydroxides (LDHs) under in-situ conditions and the catalytic mechanism can provide a thorough understanding of the structure-property relationships of many related catalysts and potentially lead to the design of new catalysts with further improved performance.

In spite of previous reports on the ex-situ crystal structure of the as-synthesized precursors of MFe LDH catalysts ${ }^{23-28}$ and insitu local structure based on X-ray absorption spectroscopy (XAS) measurements ${ }^{3,4,12,29-32}$, little is known about the long-range crystal structures of the catalytically active phase under OER conditions. As a result, most proposals regarding the in-situ crystal structures of $\mathrm{NiFe}$ and $\mathrm{CoFe} \mathrm{LDHs}$ under OER conditions are indirectly inferred from the crystal structures of the host $\mathrm{Ni}$ and $\mathrm{Co}$ oxyhydroxides, respectively. More specifically, for $\mathrm{NiFe}$ $\mathrm{LDH}$, a $\gamma$-NiOOH-type phase, in which water and cations are intercalated between layers ${ }^{28}$, has long been speculated ${ }^{4,5,24,25,33}$. However, no direct evidence has been observed to confirm this hypothesis, as previous in-situ structural studies could not provide the characteristic interlayer spacing that can be used to differentiate between the $\gamma$-NiOOH-type phase and other common phases, such as the anhydrous $\beta$-NiOOH-type phase ${ }^{28}$. For $\mathrm{CoFe} \mathrm{LDH}$, in analogy to NiFe LDH, a transformation to a $\gamma$ $\mathrm{NiOOH}$-type phase can be hypothesized under OER conditions. However, there is no analogous $\gamma-\mathrm{CoOOH}$ phase with species intercalated between layers; the other two known $\beta-\mathrm{CoOOH}$ and $\mathrm{CoO}_{2}$ phases show no intercalation ${ }^{34}$. As a consequence, a Fedoped $\beta$ - $\mathrm{CoOOH}$ has been proposed as the active phase of $\mathrm{CoFe}$ LDH under OER conditions ${ }^{11,12}$.

Density functional theory (DFT) calculations allow us to examine all of the above hypotheses and to extract atomic-scale details by screening suitable candidate phases and comparing their relative stability with that of known phases. Although significant efforts have been made, particularly on the modeling of the electronic structure effects and catalytic mechanism of $\mathrm{Ni}$ -

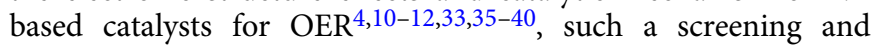
comparison has not yet been rigorously carried out because of the structural complexity of the active phases. Indeed, even the atomic-scale structure of the $\gamma-\mathrm{NiOOH}$ phase itself is still unclear ${ }^{4,33,38}$. The lack of these atomic-scale details has, in turn, made it highly challenging to choose appropriate models for DFT-based mechanistic studies ${ }^{4,38,41}$. Hence, a variety of structures have been employed in the modeling, including those that resemble as-synthesized precursor phases ${ }^{37}, \mathrm{NiO}^{38}$, twodimensional single layer (oxy)hydroxides ${ }^{35,39}, \quad \beta-\mathrm{MOOH}$ analogs $s^{4,10-12,42}$, and $\gamma$-NiOOH analogs $33,40,43-45$ with or without Fe dopants. Although significant efforts have been made to explain the high activity of MFe LDHs, the diversity of studies suggests that large uncertainties exist concerning the relationship between the active site structure and the catalytic mechanism. This is because the predicted activity of the catalysts is highly sensitive to, and is an ensemble of, the geometrical structure $^{46,47}$ and electronic structure (oxidation state) ${ }^{48,49}$ of the active site, as well as non-covalent interactions originating from bulk crystal structure ${ }^{50,51}$, the steady state of the surface configuration ${ }^{52,53}$, and the electronic structure methods used in the calculations $s^{54-56}$. These uncertainties, resulting from an incomplete consideration of this ensemble of factors, have hindered the mechanistic understanding of the high activity of NiFe and CoFe LDHs for the OER, which further hampers the prediction of new catalysts with improved performance.

Herein, we combine electrochemical measurements with operando wide-angle X-ray scattering (WAXS) and XAS data, as well as ab initio molecular dynamic simulations and a synergistic DFT approach that was benchmarked specifically for the strongly correlated $\mathrm{Fe}, \mathrm{Co}$, and $\mathrm{Ni}$ oxides and (oxy)hydroxides ${ }^{55}$, to unravel and contrast the crystal structures and electrocatalytic OER mechanisms of the active phases of NiFe and CoFe LDH catalysts. We provide the first direct atomic-scale evidence that, under OER conditions, both NiFe and CoFe LDHs transform from the as-prepared $\alpha$-phase to a deprotonated $\gamma$-phase. The oxidative phase transitions are characterized by $\sim 8 \%$ contractions in both the in-plane lattice constant and the interlayer distance, which are induced by the oxidation of $\mathrm{Fe}$ and $\mathrm{M}(\mathrm{Ni}, \mathrm{Co})$, and by the anion-to-cation switching of intercalated ions, respectively. We then adopt the in-situ identified $\gamma$-phases to study the OER mechanism through DFT-based calculations. The calculated surface phase diagrams indicate that surface $\mathrm{O}$ sites are saturated with $\mathrm{H}$ by forming bridge $\mathrm{OH}$, and undercoordinated metal sites are saturated with atop $\mathrm{OH}$ under OER conditions. These structures, and the associated reaction free energies, suggest that the OER proceeds via a Mars van Krevelen mechanism, starting with the oxidation of bridge $\mathrm{OH}$ at the $\mathrm{Fe}-\mathrm{M}$ reaction centers $(\mathrm{M}=\mathrm{Ni}$ or $\mathrm{Co})$ to form O-bridged Fe-M moieties. The flexible electronic structure of the Fe site and its synergy with the nearestneighbor $\mathrm{M}$ sites through the formation of the O-bridged $\mathrm{Fe}-\mathrm{M}$ reaction centers fundamentally accounts for the high OER activity of MFe oxyhydroxides due to the stabilization of OER intermediates that are unfavorable on pure M-M centers and single $\mathrm{Fe}$ sites. Our combined operando experimental and DFT computational approach thus provides a consistent atomic-scale explanation for the high OER activity of the MFe LDHs.

\section{Results}

Electrochemical oxygen evolution and surface redox chemistry. We studied the redox chemistry of NiFe LDH and CoFe LDH (M: $\mathrm{Fe}=\sim 3: 1)$ using cyclic and linear sweep voltammetry (CV and LSV) and compared their OER performance with that of their $\mathrm{Fe}$ free hydroxide analogs, including $\beta-\mathrm{Ni}(\mathrm{OH})_{2}$ and $\beta-\mathrm{Co}(\mathrm{OH})_{2}$. LSV curves (Fig. 1a) indicated that OER overpotentials at $10 \mathrm{~mA}$ $\mathrm{cm}^{-2}$ are $+348 \mathrm{mV}$ and $+404 \mathrm{mV}$ for NiFe LDH and CoFe LDH, respectively, which makes them among the most active electrocatalysts in alkaline conditions. $\mathrm{NiFe}$ and $\mathrm{CoFe} \mathrm{LDHs}$ also exhibited substantially higher catalytic activity than the hydroxides containing only $\mathrm{Ni}$ and Co. For NiFe LDH, the overpotential is $225 \mathrm{mV}$ lower than that of $\mathrm{NiOOH}$, whereas for $\mathrm{CoFe}$ $\mathrm{LDH}$, the corresponding overpotential is $64 \mathrm{mV}$ lower than that of $\mathrm{CoOOH}$. We note that, although it is not an intrinsic metric, the overpotential measured at $10 \mathrm{~mA} \mathrm{~cm}^{-2}$ from LSV is a valid practical parameter to compare the activity trends of the catalysts ${ }^{57}$. This is confirmed by the good agreement with the trends of the intrinsic activity extracted with two distinct methods (see discussion in Supplementary Methods and Supplementary Figs. 1 

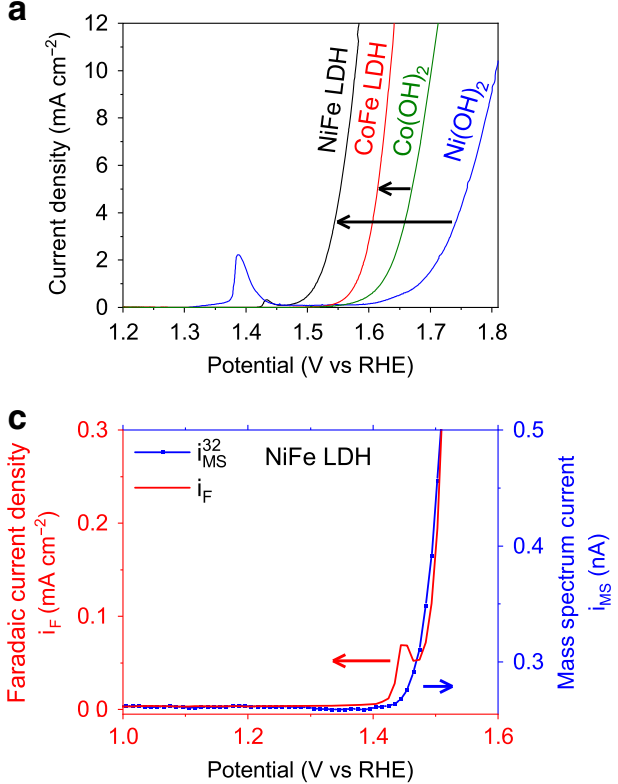

b
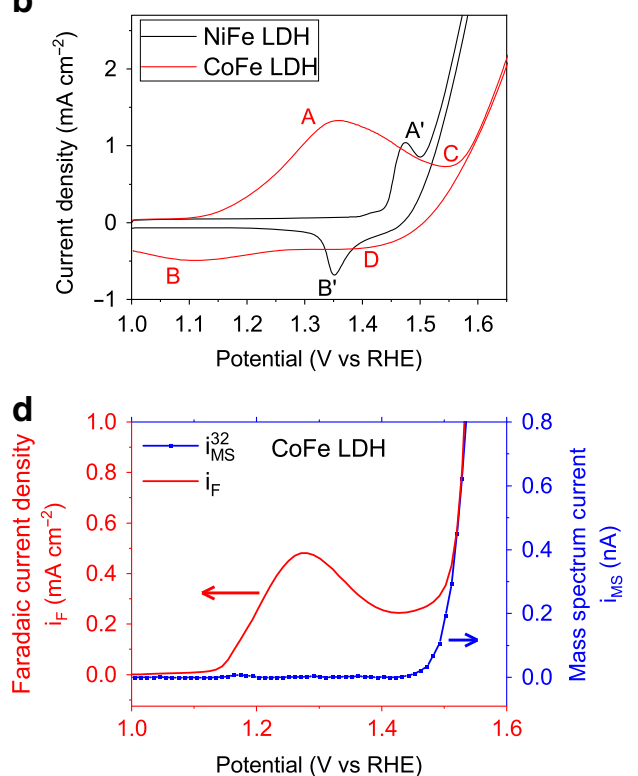

Fig. 1 Surface chemistry and OER of NiFe and CoFe LDHs. a Linear sweep voltammetry of NiFe LDH (black), CoFe LDH (red), $\beta$-Ni(OH) 2 (blue), and $\beta$-Co $(\mathrm{OH})_{2}$ (green) at a scan rate of $1 \mathrm{mV} \mathrm{s}^{-1}$ in purified $0.1 \mathrm{M} \mathrm{KOH}$ by RDE (1600 r.p.m.). Catalyst loading on GC electrodes: $0.1 \mathrm{mg}$ cm -2 . b Stable curves obtained in cyclic voltammetry of NiFe LDH (black) and CoFe LDH (red) in $0.1 \mathrm{M} \mathrm{KOH}$ in the grazing incident cell. Redox features are indicated with capital letters. c, d Differential electrochemical mass spectrometry (DEMS) of NiFe LDH (c) and CoFe LDH (d) during a linear sweep voltammetry (LSV) in 0.1 M $\mathrm{KOH}$. The faradaic current normalized by the geometric area is shown in red, whereas the mass spectrum current related to mass $m / z=32$ is shown in blue.

and 2). Also, the trend of our measurement is consistent with what was reported for electrodeposited films of similar composition ${ }^{7}$.

The CV curves in Fig. $1 \mathrm{~b}$ indicate that both $\mathrm{NiFe} \mathrm{LDH}$ and $\mathrm{CoFe} \mathrm{LDH}$ undergo redox transitions prior to (or slightly overlapping with) the onset of the OER, as confirmed by differential electrochemical mass spectrometry (DEMS) by comparing the Faraday current and the mass spectrum current related to mass $m / z=32$ (Fig. 1c, d). For NiFe LDH, the $\mathrm{Ni}$ (II) oxidation peak at $+1.47 V_{\mathrm{RHE}}\left(\mathrm{A}^{\prime}\right)$ overlaps with the OER onset and with the corresponding reduction wave peaks at $+1.35 V_{\mathrm{RHE}}$ $\left(\mathrm{B}^{\prime}\right)$. For $\mathrm{CoFe} \mathrm{LDH}$, the main oxidation peak at $+1.35 V_{\mathrm{RHE}}(\mathrm{A})$ occurs clearly prior to any OER onset. More anodically, a second and small oxidation shoulder at around $+1.55 V_{\mathrm{RHE}}(\mathrm{C})$ overlaps with the OER. The broad peaks B at $+1.1 V_{\mathrm{RHE}}$ and D at $+1.4 V_{\mathrm{RHE}}$ constitute the corresponding reduction waves on the cathodic scan, respectively. These redox features, in turn, provide strong evidence that the active phases for OER are not the as-synthesized phases (characterized in the Supplementary Methods and Supplementary Fig. 3).

Tracking structural transformations during activation. To follow the phase transition of the catalysts from their assynthesized precursor state into the catalytically active states, synchrotron-based operando WAXS analysis was employed. Insitu WAXS measurements were taken in $0.1 \mathrm{M} \mathrm{KOH}$, starting from the resting state $\left(+1 V_{\mathrm{RHE}}\right)$ of the catalysts, followed by stepping the applied potential up to $+1.7 V_{\mathrm{RHE}}$ and then back down to the resting state or even lower potentials. The scattering pattern was measured at the end of each step (i.e., Supplementary Fig. 4). The potential window ranges from values closely prior to the anodic wave of $\mathrm{M}$ (II) oxidation, reaching into the OER region and then reverting to low values to ensure the reduction to $\mathrm{M}(\mathrm{II})$. We will initially focus on the evolution of the (003) diffraction peak of the LDHs (Fig. 2a, b), which provides the characteristic interlayer distance that is absent from XAS measurements and that is central to differentiating the phases with and without intercalation of water molecules and ions. For both MFe LDHs, the evolution of the (003) peak indicates a contraction of the interlayer distance in the anodic scan and a re-expansion in the cathodic scan. The detailed interlayer distances obtained by Rietveld refinement are shown in Fig. 2c, d (additional details in Supplementary Figs. 5-9).

At the resting state and the potential before $\mathrm{M}(\mathrm{II})$ oxidation, the measured interlayer distances are $7.8 \AA$ and $7.7 \AA$ for $\mathrm{NiFe}$ and CoFe LDHs, respectively, which are typical for LDHs with intercalated water molecules and carbonate anions between the layers $^{23-28}$. As these interlayer distances resemble that of $\alpha-\mathrm{Ni}$ $(\mathrm{OH})_{2}\left(\sim 8 \AA\right.$, as proposed in the Bode's diagram $\left.{ }^{28,58}\right)$, we named this phase the $\alpha-M F e$ LDH. As soon as the potential increased above the M(II) oxidation potential, the (003) reflections shifted to shorter interlayer distances and a shoulder (Supplementary Fig. 9) started to develop at the interlayer distance of $7.2 \AA$ and $7.1 \AA$ for $\mathrm{NiFe}$ and $\mathrm{CoFe} \mathrm{LDH}$, respectively. These interlayer distances are much larger than those of the anhydrous $\beta-\mathrm{NiOOH}$ $(\sim 4.8 \AA)^{59}$ and $\beta$-CoOOH $(\sim 4.4 \AA)^{60}$ phases but are close to that of the hydrous $\gamma$-NiOOH phase (i.e., $\sim 7 \AA)^{28,59}$. In analogy to $\gamma$ $\mathrm{NiOOH}$ and previous literature ${ }^{4,24,61}$, we refer to these new phases as $\gamma$-MFe LDHs.

During the cathodic scan, the interlayer distances started to reexpand as the reduction to $\mathrm{M}(\mathrm{II})$ occurred. However, the processes depended sensitively on the nature of $\mathrm{M}$. For NiFe $\mathrm{LDH}$, the shoulder at the interlayer distance of $7.2 \AA$ ( $\gamma$-phase) disappeared at the resting state, and the peak restored to the original value of $7.8 \AA$ ( $\alpha$-phase), which indicates the reversibility of the $\alpha$-to- $\gamma$ transformation. Differently, for the CoFe LDH (Fig. 2b), the re-expansion to the original value $(7.7 \AA)$ is very limited under the resting state $\left(1 \mathrm{~V}_{\mathrm{RHE}}\right)$ and is still incomplete at lower potentials $\left(0.5 V_{\mathrm{RHE}}\right)$. The limited reversibility occurring in $\mathrm{CoFe} \mathrm{LDH}$ has also been observed during electrochemical activation treatments (Supplementary Fig. 10) and verified by 
a
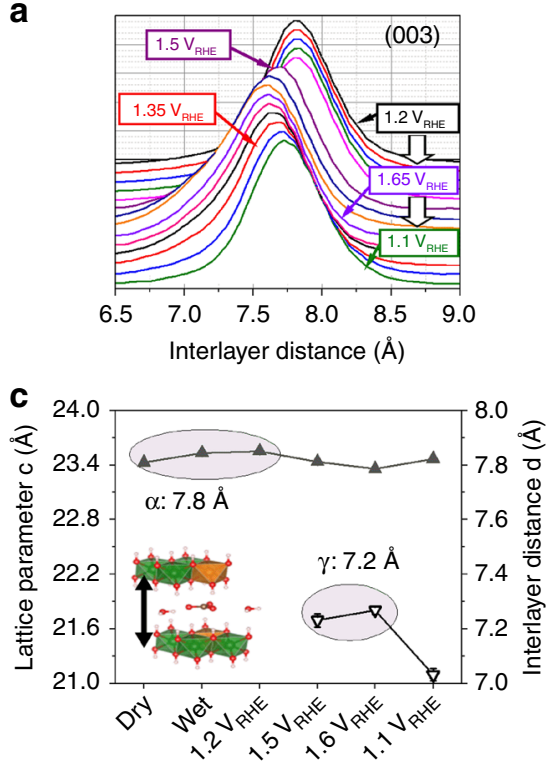

e
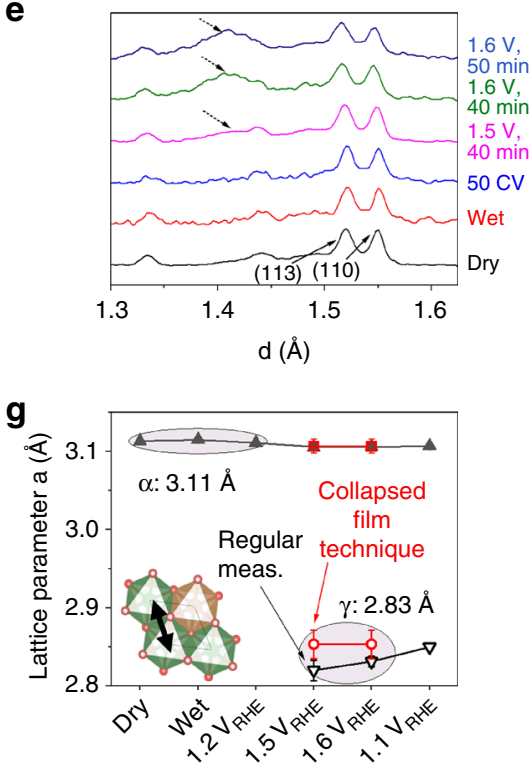

b


f
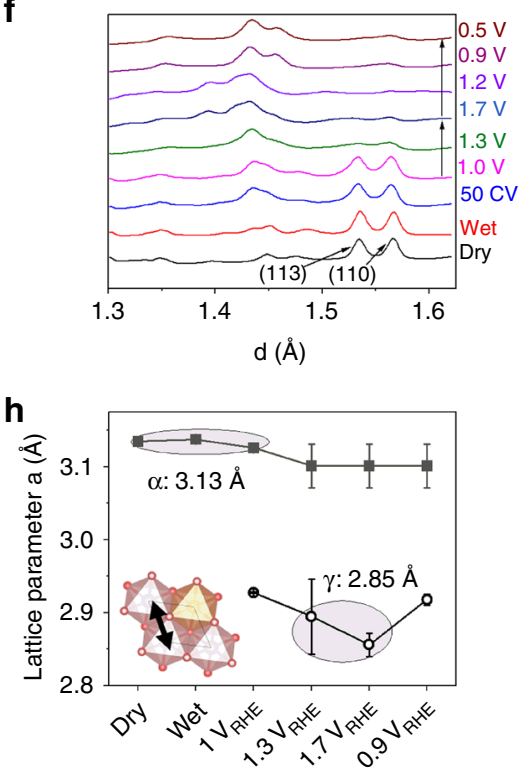

Fig. 2 The evolution of the interlayer spacing and the intralayer metal-metal distances of NiFe and CoFe LDHs from WAXS measurement.

a, b Waterfall plot of normalized and background-subtracted (003) peak obtained during in-situ WAXS in 0.1 M KOH and potential steps for NiFe LDH (a) and $\mathrm{CoFe} L D H(\mathbf{b})$. c, d Interlayer distances for NiFe LDH (c) and CoFe LDH (d) obtained by by Rietveld refinement. Full and open symbols are used for different phases. The error bars represent the SE provided by Topas. e, f In-situ WAXS patterns for $d$-values close to the (110) peak of NiFe LDH (e) and CoFe LDH (f) under various conditions. For NiFe LDH, the WAXS patterns at the reported potentials have been obtained by the collapsed film technique. In $\mathbf{e}$, the dashed arrows point to the feature associated to the $\gamma$-phase. $\mathbf{g}$, $\mathbf{h}$ Lattice parameter a, corresponding to the intralayer metal-metal distance in $\mathrm{NiFe}$ LDH (g) and CoFe LDH (h) obtained by Rietveld refinement. Full and open symbols are used for different phases. Error bars represent SD provided by Topas for the refined parameters.

ex-situ soft X-ray XAS (sXAS) (Supplementary Figs. 11 and 12). In addition, Co-based hydroxides also have shown irreversible behavior in the literature 32 .

After clarifying the catalytically active phases under OER condition via the (003) reflection, we now turn to the (110) reflection representing the in-plane lattice constants (Fig. 2e, f and Supplementary Fig. 13). As the (110) reflection is much weaker than the (003), and broadened under OER conditions, extracting exact lattice parameters is non-trivial. Nonetheless, our Rietveld refinement revealed an unambiguous trend toward shorter metal-metal distances, from $\sim 3.1 \AA$ to $\sim 2.85 \AA$ upon $\alpha$-to- $\gamma$ phase transitions for both NiFe and CoFe LDHs (Fig. 2g, h). This trend agrees well with the contraction of the local metal-O and metal-metal distances in previous in-situ EXAFS measurements ${ }^{3,4,12,29-32}$. Thus, there are contractions on both interlayer distances and in-plane bonds upon the $\alpha$-to- $\gamma$ phase transition.

We note that, similar to what has been observed in previous measurements with in-situ XAS ${ }^{31,62}$ and Mössbauer spectroscopy on NiFe-based oxyhydroxides ${ }^{63}$, only a fraction of MFe LDHs in our operando WAXS measurements undergo phase transitions under OER potentials, although the fraction is higher for CoFe than NiFe LDH (Supplementary Fig. 9). The incomplete phase transition is likely because some nanoplates in the catalyst film are not electrochemically accessible, e.g., not in contact with the 
electrolyte or with the external electrical circuit (see Supplementary Information for detailed discussion and Supplementary Figs. 14 and 15). This incompleteness makes the quantitative interpretation of XAS data challenging, as the measured local structures and electronic structures are weighted averages of the two phases. Thus, the ensemble-averaged structural parameters and the electronic structure do not necessarily reflect the actual crystal structure parameters and the electronic structure of a specific phase, but strongly depend on the ratio of the $\alpha-$ to $^{-} \gamma$ phase transition. This issue is known for unsupported NiFe (oxy)hydroxide nanocataylsts ${ }^{31}$, and confirmed by our operando XAS (see Supplementary Methods, Supplementary Figs. 16-25, and Supplementary Tables 1-4). Therefore, what sets the present operando WAXS measurements apart from other ensembleaveraging approaches is their ability to probe both intrinsic local and longer-range geometric effects of specific phases, providing essential information for the identification of the active phase under OER which cannot be achieved by the experimental techniques that solely provide average local structure information. This intrinsic structural information can serve as the reference for DFT calculations to study atomic-scale geometric structures and the intrinsic electronic structure of $\gamma$-MFe LDHs, which can in turn be further employed to study the catalytic mechanism for OER. We note that, while the $\gamma$ phase is the focus of the study, a consistent measurement of the a phase is important for establishing a general picture regarding the completeness and the reversibility of the phase transition.

\section{Geometric and electronic structures from DFT calculations.} Following the order in the above experimental section, we begin by discussing the as-prepared MFe phases ( $\mathrm{M}: \mathrm{Fe}=3: 1, \alpha-\mathrm{MFe}$ LDHs). DFT calculations indicate that $\alpha-\mathrm{MFe} \mathrm{LDHs}$ adopt the structure of hydrotalcite $\left(\mathrm{Mg}_{6} \mathrm{Al}_{2} \mathrm{CO}_{3}(\mathrm{OH})_{16} \cdot 4 \mathrm{H}_{2} \mathrm{O}\right)$, which is the archetypical LDH material with its characteristic three layer rhombohedral structure. Hydrotalcite formation is favorable from the component (oxy)hydroxides $\left(\mathrm{FeOOH}, \mathrm{M}(\mathrm{OH})_{2}\right.$ ), water (in electrolyte), and $\mathrm{CO}_{2}$ (in atmosphere) (Supplementary Figs. 2628 and Supplementary Table 5), which highlights the reliability of the present calculations. In these $\mathrm{M}_{6} \mathrm{Fe}_{2} \mathrm{CO}_{3}(\mathrm{OH})_{16} \cdot 4 \mathrm{H}_{2} \mathrm{O}$ structures, $\mathrm{Fe}^{3+}$ ions are separated by $\mathrm{M}^{2+}$ cations within the layer, and the $\mathrm{H}_{2} \mathrm{O}$ and $\mathrm{CO}_{3}{ }^{2-}$ ions are intercalated between layers in a flat configuration, interconnected through hydrogen bonds. The intercalated species are further connected with the $\mathrm{M}_{0.75} \mathrm{Fe}_{0.25}(\mathrm{OH})_{2}$ sheets by accepting hydrogen bonds from the $\mathrm{OH}$ terminations of the sheets (see Fig. 3). The calculated interlayer distances are $7.7 \AA$ for both NiFe and CoFe LDHs, which is fully consistent with the measured distances of $7.7 \AA-7.8 \AA$. The calculated in-plane lattice constants are $3.10 \AA$ and $3.15 \AA$ for $\mathrm{NiFe}$ and CoFe LDHs, respectively, which also fully agree with the measured WAXS values (3.11 $\AA$ and $3.13 \AA$, respectively; Fig. 2 ).

To identify the catalytically active phases of MFe LDHs under OER conditions, we first calculated a series of structures and configurations of $\gamma-\mathrm{NiOOH}$ with seven possible nominal oxidation states of $\mathrm{Ni}$, varying from $3+$ to $4+$, and various amounts of water molecules and ions intercalated through abinitio molecular dynamics (AIMD) simulations (see Fig. 3a, b). We then used the most plausible $\gamma-\mathrm{NiOOH}$ as the basis to study the possible configuration of $\gamma$-MFe LDHs. Among the structures considered, a phase with 4 water molecules and $2 \mathrm{~K}^{+}$cations intercalated between $\mathrm{M}_{6} \mathrm{Fe}_{2} \mathrm{O}_{16}$ layers is the most plausible phase under OER conditions. This conclusion is suggested by the favorable formation energies from its components ((hydroxy) oxides, water, and cations in the electrolyte, see Supplementary Table 5), and by the stability under OER conditions (see Fig. 3). The interlayer distances and the in-plane lattice constants are
$7.18 \AA$ and $2.84 \AA$, respectively, for both $\gamma$-type NiFe and CoFe $\mathrm{LDH}$ phases. These values are in excellent agreement with the measured values during OER of the MFe $\gamma$-phases: 7.1-7.2 $\AA$ and $\sim 2.85 \AA$, respectively. We note that the anhydrous phases with similar overall oxidation state $\left(\mathrm{M}_{0.75} \mathrm{Fe}_{0.25} \mathrm{OOH}_{0.25}\right.$ and $\mathrm{M}_{0.75} \mathrm{Fe}_{0.25} \mathrm{O}_{2}$; see Supplementary Table 6) exhibit a similar in-plane lattice constant, yet the interlayer distance is $\sim 4.6 \AA$. The similarity in the in-plane lattice constants of these two distinct phases strongly suggests that local metal-metal distance alone is insufficient to accurately identify the crystal phase present under OER condition. This fact underscores that for the present catalyst systems, the operando scattering analysis is the best technique for identifying the 3D structure of the catalytically active phases.

As described above, DFT calculations indicate that under OER conditions, MFe LDHs transform from the as-synthesized phase with the stoichiometry $\mathrm{M}_{6} \mathrm{Fe}_{2} \mathrm{CO}_{3}(\mathrm{OH})_{16} \cdot 4 \mathrm{H}_{2} \mathrm{O}$ to the $\gamma$-phase with the stoichiometry $\mathrm{M}_{6} \mathrm{Fe}_{2} \mathrm{~K}_{2} \mathrm{O}_{16} \cdot 4 \mathrm{H}_{2} \mathrm{O}$. We note that, consistent with previous measurement with Raman spectroscopy ${ }^{64}$, there are no hydroxyl groups in $\mathrm{M}_{0.75} \mathrm{Fe}_{0.25} \mathrm{O}_{2}$ layers in the $\gamma$-phase. The deprotonation of the hydroxyls of the a-phase, in turn, breaks the hydrogen bonds that exist between them and the carbonate anions and makes the intercalation of the latter highly unfavorable. Thus, $\mathrm{CO}_{3}{ }^{2-}$ ions are expelled and $\mathrm{K}^{+}$ions are intercalated from the electrolyte during the $\alpha-\gamma$ phase transition. $\mathrm{K}^{+}$ions connect $\mathrm{M}_{0.75} \mathrm{Fe}_{0.25} \mathrm{O}_{2}$ sheets by forming O-K-O ionic bonds in the form of zigzag chains. The channels between the zigzag $\mathrm{K}^{+}$chains are filled with water molecules to fully saturate the remaining oxygen atoms in the $\mathrm{M}_{0.75} \mathrm{Fe}_{0.25} \mathrm{O}_{2}$ layers through the formation of $\mathrm{O}-\mathrm{HOH}^{-} \mathrm{O}$ hydrogen bonds (see Fig. 3). Based on the intrinsic magnetic moment ${ }^{39}, \mathrm{M}$ cations are in mixed $3+$ and $4+$ oxidation states (see Supplementary Figs. 29 and 30, and Supplementary Table 5), which is consistent with the average oxidation states in the range of 3.0-3.7 that have been reported in the literature based on XAS measurements ${ }^{4,12,31,32,61,65}$. It is worth noting that, for the cases of incomplete phase transition, the measured oxidation state is a weighted average of $2+, 3+$, and $4+$. For $\gamma$-NiFe LDH, consistent with a previous assignment based on operando Mössbauer spectroscopy studies $25,63,66, \mathrm{Fe}$ cations are in a $4+$ oxidation state (see Supplementary Fig. 29 and Supplementary Table 5). We note that, in addition to $\mathrm{Fe}^{4+}$, higher $\mathrm{Fe}$ oxidation states have already been reported in the literature ${ }^{67-69}$. We will show in the reaction mechanism study below that the flexible oxidation state of the $\mathrm{Fe}$ site, and its synergy with $\mathrm{M}$ sites, are responsible for the high catalytic activity of MFe LDHs. Based on the energetics (see Fig. 3), the formation probability of the $\gamma$ phase that we screened, $\mathrm{K}_{1 / 4}\left(\mathrm{H}_{2} \mathrm{O}\right)_{1 / 2} \mathrm{MO}_{2}$, is over three orders of magnitude higher than the $\gamma-\mathrm{NiOOH}$ analog $\mathrm{K}_{1 / 3}\left(\mathrm{H}_{2} \mathrm{O}\right)_{2 / 3} \mathrm{MO}_{2}$ used in previous studies ${ }^{70}$. As the activity is sensitive to noncovalent interactions induced by the bulk structure and the electronic structure, in addition to the geometric structure and electronic structure of the active site, the $\gamma$-MFe phase is used in the study of the OER mechanism below. We note that, because of the characteristic stoichiometry of the layer and atomic-scale details of the intercalated species, the $\gamma$ phase cannot be obtained by simply introducing various amounts of water molecules and cations into the interlayer space of the $\beta-\mathrm{MOOH}$ analogs used in the literature. Further, as we demonstrate below, correct determination of the OER mechanism requires not only an accurate treatment of the bulk catalyst structure, but also a complete consideration of all key factors that have been missed in previous models, including the geometry, oxidation states, and adsorbate coverages on the catalyst surface.

The catalytic oxygen evolution reaction mechanism. Beginning with the elucidated bulk structures described above, we evaluated 

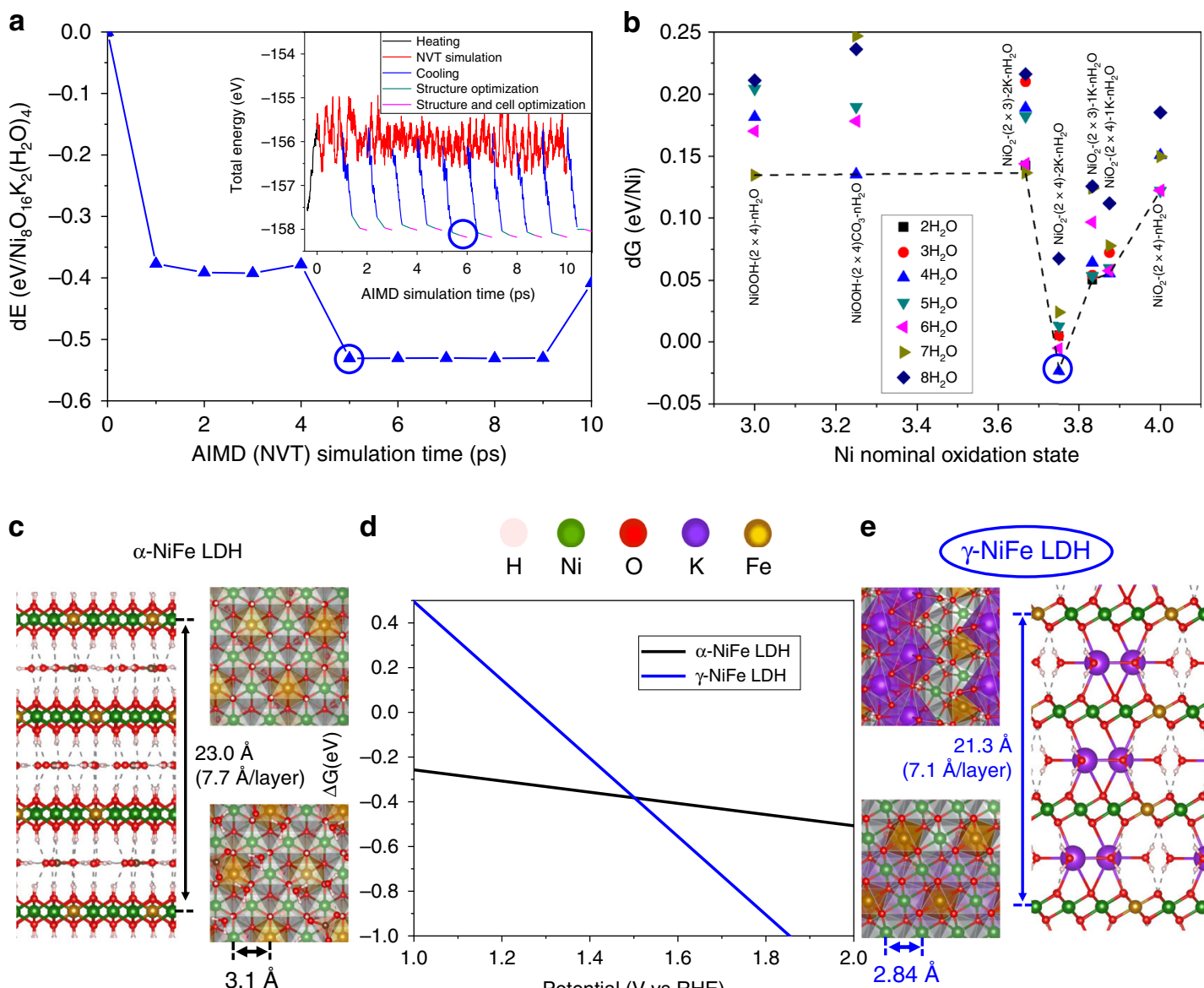

d

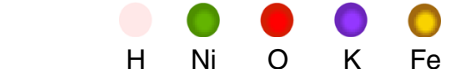

e

$\mathrm{H} \quad \mathrm{Ni} \quad \mathrm{O} \quad \mathrm{K} \quad \mathrm{Fe}$

$\gamma-\mathrm{NiFe}$ LDH

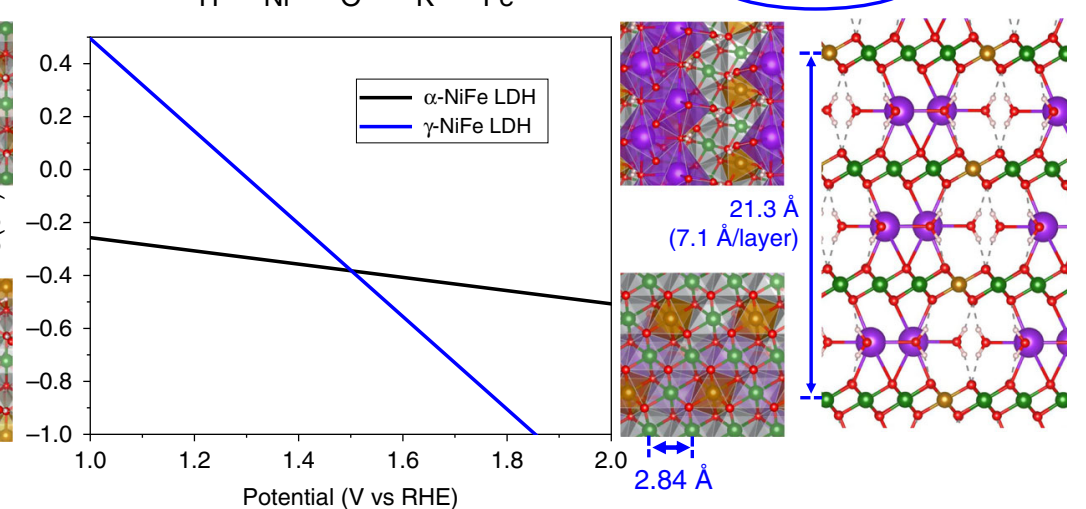

Fig. 3 Screening process, structures, and stability (phase diagram) of NiFe $\mathbf{L D H}$. a The relative energy of $\boldsymbol{\gamma}-\mathrm{NiOOH}\left(\mathrm{Ni}_{8} \mathrm{O}_{16} \mathrm{~K}_{2} \cdot 4 \mathrm{H}_{2} \mathrm{O}\right)$ at each picosecond of the AIMD simulation, which is used to screen the most stable configuration (at the 5th ps) of this specific stoichiometry. The inset is the energy evolution during the entire AIMD simulation. $\mathbf{b}$ Free energy of formation of a series of possible $\gamma$ - $\mathrm{NiOOH}$ structures with various amounts of water and ions intercalated between the $\mathrm{NiOOH}$ or $\mathrm{NiO}_{2}$ layers. Each point is based on the most stable configuration of an $\mathrm{AIMD}$ simulation. For example, $\mathrm{NiO}_{2}-(2 \times 4)$ $-2 \mathrm{~K}-4 \mathrm{H}_{2} \mathrm{O}$ is from the 5 th ps simulation of $\mathrm{Ni}_{8} \mathrm{O}_{16} \mathrm{~K}_{2} \cdot 4 \mathrm{H}_{2} \mathrm{O}$ in $\mathrm{A}$, which is then used to study the possible configuration of $\gamma-\mathrm{NiFe} \mathrm{LDH}$. c Side, top, and bottom views of the $\alpha-\mathrm{NiFe} L D H ; \boldsymbol{d}$ stability of $\alpha$ - and $\gamma-\mathrm{NiFe} L D H$; e side, top, and bottom views of the $\gamma$-NiFe LDH. The structural parameters of $\alpha-$ and $\gamma$-NiFe LDH are also given.

the steady state of the (01-10) surface of $\gamma$-NiOOH, $\gamma$-NiFe $\mathrm{LDH}$, and $\gamma$-CoFe LDH through surface phase diagrams, then calculated the reaction free energy diagram of oxygen redox $\left(4 \mathrm{OH}^{-}+\right.$ $* \leftarrow 33 \mathrm{OH}^{-}+\mathrm{OH}^{*}+\mathrm{e}^{-} \leftarrow \rightarrow 2 \mathrm{OH}^{-}+\mathrm{O}^{*}+\mathrm{H}_{2} \mathrm{O}+2 \mathrm{e}^{-} \rightarrow \mathrm{OH}^{-}$ $+\mathrm{OOH}^{*}+\mathrm{H}_{2} \mathrm{O}+3 \mathrm{e}^{-} \rightarrow \mathrm{O}_{2}+2 \mathrm{H}_{2} \mathrm{O}+4 \mathrm{e}^{-}+{ }^{*}$ ) (see Fig. 4, Supplementary Figs. 31-41, and Supplementary Tables 7-11). We have focused on the reaction of the surface oxygen species and neglected the potential involvement of lattice oxygen, because recent isotope experiments suggest that the latter is not favorable for these specific systems ${ }^{2}$. We selected the (01-10) surface, because it belongs to the family of surfaces that are exposed at the edge of catalyst sheets, and thus widely used to study the catalytic activity of layered materials $4,11,33,40,71$.

The calculated surface phase diagrams indicate that, under OER conditions, undercoordinated surface $\mathrm{O}$ sites are saturated with $\mathrm{H}$ by forming bridge $\mathrm{OH}$ species, and undercoordinated metal sites are saturated with atop $\mathrm{OH}$ when the surface is in equilibrium with the electrolyte and in steady state (see Fig. 4). Thus, we analyze the reaction free energy with a Mars van Krevelen-type mechanism, for which the reactions start from the deprotonation of the surface $\mathrm{OH}$ of the in-situ surface phase, instead of starting from $\mathrm{OH}$ adsorption, as has generally been assumed in many previous studies (to further motivate this choice, see the comparison with the reaction free energies of the conventional mechanism on two artificial surface models in the Supplementary Materials). For the Mars van Krevelen mechanism, we have found that the oxidation of two-metal coordinated bridge $\mathrm{OH}$ moieties is more favorable than that of one-metal coordinated atop $\mathrm{OH}$ due to the synergy of the two nearestneighbor metal sites in stabilizing the potential limiting OER reaction intermediates $\left(\mathrm{O}^{*}\right.$ radials) by forming an O-bridged reaction center (see Fig. 4 and Supplementary Methods for details). Thus, we have focused our discussion below on the synergistic bridge $\mathrm{OH}$ oxidation pathway.

The highest reaction free energy barriers $\left(\Delta G_{\mathrm{a}}\right)$ on $\gamma-\mathrm{NiOOH}$, $\gamma$-CoFe, and $\gamma$-NiFe oxyhydroxide surfaces are $1.90 \mathrm{eV}, 1.71 \mathrm{eV}$, and $1.68 \mathrm{eV}$ (see Fig. 4c and Supplementary Figs. 31, 33, and 38), respectively, which implies overpotentials $(\eta)$ of $0.67 \mathrm{~V}, 0.48 \mathrm{~V}$, and $0.45 \mathrm{~V}\left(\eta=\left(\Delta G_{\mathrm{a}}-1.23 \mathrm{eV}\right) / \mathrm{e}\right)$. The calculated overpotentials are semi-quantitatively consistent with the present measurements at $10 \mathrm{~mA} \mathrm{~cm}^{-2}, 0.57 \mathrm{~V}, 0.40 \mathrm{~V}$, and $0.35 \mathrm{~V}$, respectively, and with general trends in the literature. For $\gamma-\mathrm{NiOOH}$ and $\gamma-\mathrm{NiFe} \mathrm{LDH}$, $\mathrm{OH}^{*}$ deprotonation during the OER cycle has the highest free energy barrier, which forms the potential limiting step, followed by $\mathrm{OOH}^{*}$ deprotonation. On the $\gamma-\mathrm{NiOOH}$ surface, bridge $\mathrm{OH}\left(\mathrm{Ni}^{3+}-\mathrm{OH}-\mathrm{Ni}^{4+}\right)$ deprotonation at $1.90 \mathrm{~V}$ is accompanied by $\mathrm{Ni}^{3+}$ oxidation to $\mathrm{Ni}^{4+}$, as characterized by the change of Ni magnetic moment from $1 \mu_{\mathrm{B}}$ to $0 \mu_{\mathrm{B}}$. On the other hand, on 



Fig. 4 OER mechanism on the $\boldsymbol{\gamma}$-phase of MFe LDHs. a Structures of different surface phases and OER intermediates; adsorbates of surface phases are highlighted by blue circles on the sides views, and OER intermediates are differentiated by colors (yellow instead of white for hydrogen and rose instead of red for oxygen, respectively). A dashed rose circle indicates the formation of a surface $O$ vacancy. The reaction centers are highlighted by large white circles. The magnetic moments of $\mathrm{Ni}$ and Fe during OER are also given on the top views. $\mathbf{b}$ Surface phase diagram of of $\gamma-\mathrm{NiOOH}, \gamma-\mathrm{NiFe} \mathrm{LDH}$, and $\gamma$-CoFe $\mathrm{LDH}$. The representative surface phases are given in a. c Reaction free-energy diagrams for OER on $\gamma$ - NiOOH, $\gamma$-NiFe LDH, and $\gamma$-CoFe LDH; the potential limiting steps and the overpotentials are also given. $\mathbf{d}$ Volcano plot of the OER overpotential as a function of Gibbs free energies of the reaction intermediates.

the $\gamma$-NiFe $\mathrm{LDH}$ surface, bridge $\mathrm{OH}\left(\mathrm{Fe}^{4+}-\mathrm{OH}-\mathrm{Ni}^{3+}\right)$ deprotonation at $1.68 \mathrm{~V}$ is accompanied by $\mathrm{Fe}^{4+}$ oxidation (as characterized by the change of Fe magnetic moment, see Fig. 4), whereas the oxidation state of $\mathrm{Ni}$ is constant. Clearly, it is more feasible for $\mathrm{Fe}$ than $\mathrm{Ni}$ to be oxidized to a higher oxidation state, which stabilizes $\mathrm{O}^{*}$ intermediates at the Fe-Ni reaction center compared with that at the $\mathrm{Ni}-\mathrm{Ni}$ reaction center, and consequently lowers the free energy barrier of $\mathrm{OH}^{*}$ oxidation to $\mathrm{O}^{*}$, the potential limiting step. Our calculations indicate that it is also the case for the other $\gamma$-NiFe configurations with comparable energies that could coexist under the reaction conditions (see Supplementary Table 9).

It is worth noting that this stabilization effect is also valid and even stronger on single $\mathrm{Fe}$ sites as compared with single $\mathrm{Ni}$ sites $(0.73 \mathrm{eV}$ for the stabilization on single site vs. $0.22 \mathrm{eV}$ for the stabilization at the $\mathrm{Fe}-\mathrm{Ni}$ center), as also observed in previous studies $(0.4-0.5 \mathrm{eV})^{33,43}$. However, there is a fundamental difference between the synergistic stabilization through the Fe$\mathrm{Ni}$ reaction center and the stabilization through the single Fe site, with the former being over five orders of magnitude more active than the latter toward OER on NiFe LDH (see Supplementary Figs. 33 and 34). Similar synergy of two nearest-neighbor metal sites (reaction center) and flexibility of $\mathrm{Fe}$ site oxidation are also found on $\gamma$-CoFe LDH, for which the stabilization effect is so significant that bridge $\mathrm{OH}\left(\mathrm{Fe}^{4+}-\mathrm{OH}-\mathrm{Co}^{4+}\right)$ deprotonation and the accompanied $\mathrm{Fe}^{4+}$ oxidation is not the potential limiting step anymore. Instead, $\mathrm{OOH}^{*}$ deprotonation to $\mathrm{O}_{2}(\mathrm{~g})+$ vacancy (with a $1.7 \mathrm{eV}$ free-energy barrier) becomes the potential limiting step. On pure Co sites of $\gamma$-CoFe $\mathrm{LDH}, \mathrm{OOH}^{*}$ deprotonation is also the potential limiting step but with higher overpotential (at
$1.83 \mathrm{~V}$ ) because of the more unfavorable $\mathrm{O}$ vacancy formation. Therefore, in addition to the $\mathrm{O}^{*}$ intermediate, the reaction center also can stabilize $\mathrm{O}$ vacancies in CoFe LDH through synergy and the flexible electronic structure of Fe. However, the stabilization effect on the $\mathrm{O}$ vacancy formation does not seem large enough to make $\gamma$-CoFe LDH more active than $\gamma$-NiFe LDH, whereas the stabilization of $\mathrm{O}^{*}$ by the introduction of $\mathrm{Fe}$ is beneficial in both catalysts, resulting in a small difference in activity. As a consequence, the overpotentials on $\mathrm{NiFe}$ and $\mathrm{CoFe} \mathrm{LDH}$ are only modestly $(0.14-0.22 \mathrm{~V})$ higher than the optimal overpotential that is constrained by the scaling relationship (the scaling relationship of $\mathrm{OOH}^{*}$ intermediate and $\mathrm{OH}^{*}$ intermediate, which is $2.95 \mathrm{eV}$ in the present work, leads to an optimal overpotential of $0.25 \mathrm{~V})^{72}$. Such a constraint also implies that the OER overpotential of LDHs can be modestly improved by further stabilizing $\mathrm{O}^{*}$ intermediates and surface $\mathrm{O}$ vacancies at the reaction centers simultaneously, perhaps with a more redoxflexible metal than $\mathrm{Fe}$, or significantly improved by breaking the $\mathrm{OOH}^{*}$ vs. $\mathrm{OH}^{*}$ scaling relationship.

\section{Discussion}

$\mathrm{NiFe}$ and $\mathrm{CoFe} \mathrm{LDHs}$ are the archetypes of high-performing electrocatalysts for oxygen evolution in alkaline conditions. In the current work, we have identified the crystal structures of the active phase and the reaction mechanism by combining operando experiments, rigorous DFT calculations, and self-consistent mechanistic studies. We have found that, under applied anodic potentials, both $\mathrm{NiFe}$ and $\mathrm{CoFe} \mathrm{LDHs}$ transform from the 
as-prepared $\alpha$-phase to the active $\gamma$-phase. In comparison with the as-prepared phase, with an interlayer distance of $7.7 \AA$ and an in-plane lattice constant of $3.1 \AA$, the catalytically active phases are characterized by a compression of both lattice spacings to 7.1 $\AA$ and $2.8 \AA$, respectively. These values were extracted from operando WAXS measurements and are also supported by DFT calculations. Although the latter is induced by the oxidation of both $\mathrm{Fe}(\mathrm{III})$ and $\mathrm{M}(\mathrm{II})$, the former is related to the swapping of intercalated ions with $\mathrm{K}^{+}$, which is essential in identifying the crystal structure of the active phases and cannot be accessed experimentally with other local structure-based techniques. Thus, the combination of DFT and operando WAXS confirms a long speculated hypothesis regarding the crystal structure of $\mathrm{NiFe}$ LDH under OER conditions and disprove previous assumptions of the crystal structure of CoFe LDH, while, more importantly, providing key atomic-scale details of the in-situ phases for the study of the catalytic mechanism through DFT calculations. Our calculations demonstrate that OER proceeds with a Mars van Krevelen-type mechanism on these surfaces. The flexible electronic structure of the Fe sites and their synergy with the nearestneighbor $\mathrm{M}$ sites $(\mathrm{M}=\mathrm{Ni}$ or $\mathrm{Co})$ through forming O-bridged $\mathrm{Fe}-\mathrm{M}$ reaction centers stabilize OER intermediates that are unfavorable on M-M centers and pure Fe sites. This synergistic reaction center fundamentally accounts for the experimentally observed low overpotentials of MFe for OER. The present study suggests that doping oxides with additional redox-flexible metals to form active reaction centers through the synergy with nearestneighbor metal sites constitutes a general design principle for the synthesis of new OER catalysts design with improved catalytic performance.

\section{Methods}

Synthesis. NiFe LDH $(\mathrm{Ni}: \mathrm{Fe}=3.55: 1)$ was synthesized by a previously reported solvothermal route in an autoclave ${ }^{73}$. $\mathrm{CoFe} \mathrm{LDH}(\mathrm{Co}: \mathrm{Fe}=3.33: 1)$ was synthesized by using co-precipitation followed by a solvothermal treatment in an autoclave. $\mathrm{Ni}$ $(\mathrm{OH})_{2}$ was synthesized using a two-step synthesis consisting of a precipitation step and a subsequent hydrothermal treatment. $\beta-\mathrm{Co}(\mathrm{OH})_{2}$ was synthesized by a similar process as that described by $\mathrm{Ma}$ et $\mathrm{al} .{ }^{74}$ based on homogeneous precipitation. Further details are available in the Supplementary Information.

RDE measurements and DEMS. RDE electrochemical experiments were performed in a three-compartment glass cell with a rotating disk electrode (RDE, $5 \mathrm{~mm}$ in diameter of GC, Pine Instrument) and a potentiostat (Gamry) at room temperature. A Pt-mesh and a Hydroflex reversible hydrogen electrode (RHE, Gaskatel) were used as counter electrode and reference electrode, respectively. The electrolytes were prepared with $\mathrm{KOH}$ pellets (semiconductor grade, $99.99 \%$ trace metals basis, Aldrich) and MilliQ water, and were further purified 75,76 . The catalyst was deposited on the GC by drop casting from an ink based on isopropanol/water solution with Nafion as a binder. The catalyst loading was $0.1 \mathrm{mg} \mathrm{cm}^{-2}$. The detailed protocol is provided in Supplementary Methods.

DEMS measurements were performed using dual thin-layer electrochemical flow cell (see Supplementary Methods for details) with nitrogen-saturated electrolyte $0.1 \mathrm{M} \mathrm{KOH}$.

In-situ WAXS and Rietveld refinement. The electrodes used for in-situ WAXS were prepared similarly as for the RDE measurements. A home-made grazing incident cell (Supplementary Fig. 4) based on a thin-layer concept was used with a polyether ether ketone foil covering the top part of the cell as X-Ray window ${ }^{77}$. $\mathrm{KOH}(0.1 \mathrm{M})$ was used as electrolyte. In-situ WAXS experiments have been conducted at the ID31 beamline of the European Synchrotron Radiation Facility (Grenoble, France), using hard X-rays with a monochromatized beam $(60-77 \mathrm{KeV})$. The electrochemical protocol consisted in keeping the sample first at the potential of $1 V_{\text {RHE }}$ after electrolyte injection (wet condition), recording electrochemical impedance spectroscopy, conducting an activation procedure by $\mathrm{CV}$, and potential steps of $\sim 10 \mathrm{~min}$ for the regular measurements ( $40 \mathrm{~min}$ for collapsed film technique explained in the in-situ WAXS section in the Supplementary Methods) from resting state, before the $\mathrm{M}(\mathrm{II})$ oxidation ( $\mathrm{M}=\mathrm{Ni}$ or $\mathrm{Co}$ ), to OER potentials and back in the cathodic direction well below the reduction potential to M(II). The (003) and (110) peaks were fitted by Pseudo-Voigt functions in the preliminary analysis, after background subtraction. Rietveld refinement was performed on selected potentials. The hydrotalcite structure with space group R-3m was used as a model for both the
LDH materials and for both the as-prepared and oxidized phases. For full details, see the Supplementary Information.

Operando XAS. Operando XAS measurements were performed at the BL22 CLAESS beamline at ALBA light source (Barcelona, Spain) in fluorescence mode using a silicon drift diode detector. A home-made electrochemical cell was employed. A platinum mesh and leak-free $\mathrm{Ag} / \mathrm{AgCl}$ electrode were used as counter and reference electrode, respectively. The powder samples were deposited on graphite paper discs (Toray Carbon Paper TP-060, Quintech) by filtration from a slurry of the sample in ethanol containing Nafion $(0.1 \mathrm{v} / \mathrm{v} \%)$ as a binding agent. The paper discs were mounted in the operando cell so that the unmodified side was facing out, whereas the side containing the catalyst layer was in contact with the electrolyte. The electrochemical conditions were identical to those described for insitu WAXS measurements.

DFT calculation parameters. Self-consistent, periodic DFT calculations were performed with the projected augmented wave method, as implemented in the Vienna Ab-initio Simulation Package. To generate highly accurate electrochemical stability diagrams, we employ a recently developed approach ${ }^{55}$, which includes the use of a Hubbard U term, a van der Waals functional (optPBE) ${ }^{78}$, and the use of a water-based reference state for the calculations. $U$-values, which are applied to $d$-orbitals of $\mathrm{Fe}, \mathrm{Co}$, and $\mathrm{Ni}$ are taken as $2.56,3.50$, and $5.20 \mathrm{eV}$, respectively. For cell shape and volume relaxations of (hydroxy)oxide compounds, a cutoff energy of $500 \mathrm{eV}$ is used for the planewave expansion. For the calculations that do not involve cell optimization, a cutoff energy of $400 \mathrm{eV}$ is employed. Monkhorst-Pack k-point grids are used for Brillouin zone integration. $\mathrm{A}(2 \times 4 \times 1)$ and a $(2 \times 4 \times 3) \mathbf{k}$-point grid are employed for $\alpha$ - and $\gamma$-phase of LDH with R3 and R1 symmetry, respectively. For the other bulk and surface calculations, equivalent or denser $\mathbf{k}$-point grids are utilized. An orthorhombic box $(14 \times 15 \times 16) \AA^{3}$ and a single $\mathbf{k}$-point $(0.25,0.25,0.25)$ for the Brillouin zone sampling are used for gas phase species. The equilibrium geometries are obtained when the maximum atomic forces are smaller than $0.01 \mathrm{eV} / \AA$ and when a total energy convergence of $10^{-5} \mathrm{eV}$ is achieved for the electronic self-consistent field loop. AIMD simulations are performed at $400 \mathrm{~K}$ and quenched down to $0 \mathrm{~K}$ every 1 ps with a total simulation time of $10 \mathrm{ps}$. To evaluate the solvation energy of OER intermediates (see Supplementary Table 11), vacuum between the slab and the images is filled with liquid water with a thickness that is equivalent to five water bilayers. Then AIMD simulations are performed with the same protocols and time scales as that described above.

\section{Data availability.}

The data supporting the findings of this study are available within this Article and its Supplementary Information files, or from the corresponding author upon reasonable request. The Supplementary Information contains descriptions of methods, discussions on physicochemical characterization of as-prepared MFe LDH, intermediate phases, size of coherently scattering domains, operando XAS, ex-situ sXAS, and DFT calculation. It also includes Supplementary Figs. 1-41 and Supplementary Tables 1-11.

Received: 30 March 2020; Accepted: 21 April 2020;

Published online: 20 May 2020

\section{References}

1. McCrory, C. C. L. et al. Benchmarking hydrogen evolving reaction and oxygen evolving reaction electrocatalysts for solar water splitting devices. J. Am. Chem. Soc. 137, 4347-4357 (2015).

2. Roy, C. et al. Impact of nanoparticle size and lattice oxygen on water oxidation on NiFeOxHy. Nat. Catal. 1, 820-829 (2018).

3. Dresp, S. et al. Direct electrolytic splitting of seawater: activity, selectivity, degradation, and recovery studied from the molecular catalyst structure to the electrolyzer cell level. Adv. Energy Mater. 8, 1800338 (2018).

4. Friebel, D. et al. Identification of highly active $\mathrm{Fe}$ sites in $(\mathrm{Ni}, \mathrm{Fe}) \mathrm{OOH}$ for electrocatalytic water splitting. J. Am. Chem. Soc. 137, 1305-1313 (2015).

5. Trotochaud, L., Young, S. L., Ranney, J. K. \& Boettcher, S. W. Nickel-iron oxyhydroxide oxygen-evolution electrocatalysts: the role of intentional and incidental iron incorporation. J. Am. Chem. Soc. 136, 6744-6753 (2014).

6. Gong, M. et al. An advanced Ni-Fe layered double hydroxide electrocatalyst for water oxidation. J. Am. Chem. Soc. 135, 8452-8455 (2013).

7. Burke, M. S. et al. Revised oxygen evolution reaction activity trends for firstrow transition-metal (Oxy)hydroxides in alkaline media. J. Phys. Chem. Lett. 6, 3737-3742 (2015).

8. Dionigi, F. \& Strasser, P. NiFe-based (oxy)hydroxide catalysts for oxygen evolution reaction in non-acidic electrolytes. Adv. Energy Mater. 6, 1600621 (2016).

9. Hoang, T. T. H. \& Gewirth, A. A. High activity oxygen evolution reaction catalysts from additive-controlled electrodeposited $\mathrm{Ni}$ and NiFe films. ACS Catal. 6, 1159-1164 (2016). 
10. Stevens, M. B. et al. Ternary Ni-Co-Fe oxyhydroxide oxygen evolution catalysts: Intrinsic activity trends, electrical conductivity, and electronic band structure. Nano Res. 12, 2288-2295 (2019).

11. Zhang, B. et al. Homogeneously dispersed multimetal oxygen-evolving catalysts. Science 352, 333-337 (2016).

12. Enman, L. J. et al. Operando X-ray absorption spectroscopy shows iron oxidation is concurrent with oxygen evolution in cobalt-iron (oxy)hydroxide electrocatalysts. Angew. Chem. Int. Ed. 57, 12840-12844 (2018).

13. Risch, M. et al. Structural changes of cobalt-based perovskites upon water oxidation investigated by EXAFS. J. Phys. Chem. C. 117, 8628-8635 (2013).

14. Fabbri, E. et al. Dynamic surface self-reconstruction is the key of highly active perovskite nano-electrocatalysts for water splitting. Nat. Mater. 16, 925 (2017).

15. Calvillo, L. et al. Insights into the durability of Co-Fe spinel oxygen evolution electrocatalysts via operando studies of the catalyst structure. J. Mater. Chem. A 6, 7034-7041 (2018)

16. Zhang, B. W., Lui, Y. H., Zhou, L., Tang, X. H. \& Hu, S. An alkaline electroactivated $\mathrm{Fe}-\mathrm{Ni}$ phosphide nanoparticle-stack array for high-performance oxygen evolution under alkaline and neutral conditions. J. Mater. Chem. A 5, 13329-13335 (2017).

17. Kun, X. et al. Dual electrical-behavior regulation on electrocatalysts realizing enhanced electrochemical water oxidation. Adv. Mater. 28, 3326-3332 (2016).

18. Yongqi, Z. et al. Rapid synthesis of cobalt nitride nanowires: highly efficient and low-cost catalysts for oxygen evolution. Angew. Chem. Int. Ed. 55, 8670-8674 (2016).

19. Yu, C. et al. An electrocatalyst with anti-oxidized capability for overall water splitting. Nano Res. 11, 3411-3418 (2018).

20. Zhang, J.-Y. et al. Rational design of cobalt-iron selenides for highly efficient electrochemical water oxidation. ACS Appl. Mater. Interfaces 9, 33833-33840 (2017).

21. Fabbri, E. et al. Dynamic surface self-reconstruction is the key of highly active perovskite nano-electrocatalysts for water splitting. Nat. Mater. 16, 925-92 (2017).

22. Zhuang, Z., Sheng, W. \& Yan, Y. Synthesis of monodispere Au@Co3O4 coreshell nanocrystals and their enhanced catalytic activity for oxygen evolution reaction. Adv. Mater. 26, 3950-3955 (2014).

23. Ma, R., Liang, J., Liu, X. \& Sasaki, T. General insights into structural evolution of layered double hydroxide: underlying aspects in topochemical transformation from brucite to layered double hydroxide. J. Am. Chem. Soc. 134, 19915-19921 (2012).

24. Demourguesguerlou, L., Braconnier, J. J. \& Delmas, C. Iron-substituted nickel oxyhydroxides and hydroxides obtained by Chimie-Douce. J. Solid State Chem. 104, 359-367 (1993).

25. Demourguesguerlou, L., Fournes, L. \& Delmas, C. On the iron oxidation-state in the iron-substituted gamma-nickel oxyhydroxides. J. Solid State Chem. 114, 6-14 (1995).

26. Batchellor, A. S., Kwon, G., Laskowski, F. A. L., Tiede, D. M. \& Boettcher, S. W. Domain structures of $\mathrm{Ni}$ and $\mathrm{NiFe}$ (oxy)hydroxide oxygen-evolution catalysts from X-ray pair distribution function analysis. J. Phys. Chem. C. 121, 25421-25429 (2017).

27. Hunter, B. M., Hieringer, W., Winkler, J. R., Gray, H. B. \& Muller, A. M. Effect of interlayer anions on [NiFe]-LDH nanosheet water oxidation activity. Energy Environ. Sci. 9, 1734-1743 (2016).

28. Doyle, R. L., Godwin, I. J., Brandon, M. P. \& Lyons, M. E. G. Redox and electrochemical water splitting catalytic properties of hydrated metal oxide modified electrodes. Phys. Chem. Chem. Phys. 15, 13737-13783 (2013).

29. Görlin, M. et al. Oxygen evolution reaction dynamics, Faradaic charge efficiency, and the active metal redox states of $\mathrm{Ni}-\mathrm{Fe}$ oxide water splitting electrocatalysts. J. Am. Chem. Soc. 138, 5603-5614 (2016).

30. Bates, M. K., Jia, Q., Doan, H., Liang, W. \& Mukerjee, S. Charge-transfer effects in $\mathrm{Ni}-\mathrm{Fe}$ and $\mathrm{Ni}-\mathrm{Fe}-\mathrm{Co}$ mixed-metal oxides for the alkaline oxygen evolution reaction. ACS Catal. 6, 155-161 (2016).

31. Gorlin, M. et al. Tracking catalyst redox states and reaction dynamics in $\mathrm{Ni}-\mathrm{Fe}$ oxyhydroxide oxygen evolution reaction electrocatalysts: the role of catalyst support and electrolyte pH. J. Am. Chem. Soc. 139, 2070-2082 (2017).

32. Smith, R. D. L. et al. Spectroscopic identification of active sites for the oxygen evolution reaction on iron-cobalt oxides. Nat. Commun. 8, 2022 (2017).

33. Xiao, H., Shin, H. \& Goddard, W. A. Synergy between Fe and Ni in the optimal performance of $(\mathrm{Ni}, \mathrm{Fe}) \mathrm{OOH}$ catalysts for the oxygen evolution reaction. Proc. Natl Acad. Sci. USA 115, 5872-5877 (2018).

34. Bajdich, M., Garcia-Mota, M., Vojvodic, A., Norskov, J. K. \& Bell, A. T. Theoretical investigation of the activity of cobalt oxides for the electrochemical oxidation of water. J. Am. Chem. Soc. 135, 13521-13530 (2013).

35. Zaffran, J. \& Toroker, M. C. Understanding the oxygen evolution reaction on a two-dimensional $\mathrm{NiO}_{2}$ catalyst. ChemElectroChem 4, 2764-2770 (2017)

36. Nagli, M. \& Caspary Toroker, M. Communication: nickel hydroxide as an exceptional deviation from the quantum size effect. J. Chem. Phys. 149, 141103 (2018).
37. Zhang, J. et al. Single-atom Au/NiFe layered double hydroxide electrocatalyst probing the origin of activity for oxygen evolution reaction. J. Am. Chem. Soc 140, 3876-3879 (2018).

38. Diaz-Morales, O., Ledezma-Yanez, I., Koper, M. T. M. \& Calle-Vallejo, F. Guidelines for the rational design of Ni-based double hydroxide electrocatalysts for the oxygen evolution reaction. Acs Catal. 5, 5380-5387 (2015).

39. Goldsmith, Z. K. et al. Characterization of NiFe oxyhydroxide electrocatalysts by integrated electronic structure calculations and spectroelectrochemistry. Proc. Natl Acad. Sci. USA 114, 3050-3055 (2017).

40. Li, Y. F. \& Selloni, A. Mechanism and activity of water oxidation on selected surfaces of pure and Fe-doped NiOx. Acs Catal. 4, 1148-1153 (2014).

41. Tripkovic, V., Hansen, H. A. \& Vegge, T. From 3D to 2D Co and Ni oxyhydroxide catalysts: elucidation of the active site and influence of doping on the oxygen evolution activity. ACS Catal. 7, 8558-8571 (2017).

42. Martirez, J. M. P. \& Carter, E. A. Unraveling oxygen evolution on iron-doped beta-nickel oxyhydroxide: the key role of highly active molecular-like sites. J. Am. Chem. Soc. 141, 693-705 (2019).

43. Shin, H., Xiao, H. \& Goddard, W. A. In silico discovery of new dopants for Fedoped $\mathrm{Ni}$ oxyhydroxide $(\mathrm{Nil}-\mathrm{xFexOOH})$ catalysts for oxygen evolution reaction. J. Am. Chem. Soc. 140, 6745-6748 (2018).

44. Zaffran, J. et al. Influence of electrolyte cations on $\mathrm{Ni}(\mathrm{Fe}) \mathrm{OOH}$ catalyzed oxygen evolution reaction. Chem. Mater. 29, 4761-4767 (2017).

45. Baker, J. G. et al. The role of aluminum in promoting $\mathrm{Ni}-\mathrm{Fe}-\mathrm{OOH}$ electrocatalysts for the oxygen evolution reaction. ACS Appl Energ. Mater. 2, 3488-3499 (2019)

46. Liu, J.-X., Su, H.-Y., Sun, D.-P., Zhang, B.-Y. \& Li, W.-X. Crystallographic dependence of $\mathrm{CO}$ activation on cobalt catalysts: HCP versus FCC. J. Am. Chem. Soc. 135, 16284-16287 (2013).

47. Li, H., Li, Y., Koper, M. T. M. \& Calle-Vallejo, F. Bond-making and breaking between carbon, nitrogen, and oxygen in electrocatalysis. J. Am. Chem. Soc. 136, 15694-15701 (2014).

48. Hammer, B. \& Nørskov, J. K. in Advances in Catalysis 45, 71-129 (Academic Press, Inc., 2000).

49. Lee, Y.-L., Kleis, J., Rossmeisl, J., Shao-Horn, Y. \& Morgan, D. Prediction of solid oxide fuel cell cathode activity with first-principles descriptors. Energy Environ. Sci. 4, 3966-3970 (2011).

50. Strmcnik, D. et al. The role of non-covalent interactions in electrocatalytic fuel-cell reactions on platinum. Nat. Chem. 1, 466-472 (2009).

51. Li, H., Xiao, J., Fu, Q. \& Bao, X. Confined catalysis under two-dimensional materials. Proc. Natl Acad. Sci. USA 114, 5930-5934 (2017).

52. Reuter, K., Frenkel, D. \& Scheffler, M. The steady state of heterogeneous catalysis, studied by first-principles statistical mechanics. Phys. Rev. Lett. 93, 116105 (2004)

53. Wang, S., Vorotnikov, V. \& Vlachos, D. G. Coverage-induced conformational effects on activity and selectivity: hydrogenation and decarbonylation of furfural on Pd(111). ACS Catal. 5, 104-112 (2015).

54. Hensley, A. J. R. et al. DFT-based method for more accurate adsorption energies: an adaptive sum of energies from RPBE and vdW density functionals. J. Phys. Chem. C. 121, 4937-4945 (2017).

55. Zeng, Z. et al. Towards first principles-based prediction of highly accurate electrochemical Pourbaix diagrams. J. Phys. Chem. C. 119, 18177-18187 (2015).

56. Zaffran, J. \& Toroker, M. C. Benchmarking density functional theory based methods to model NiOOH material properties: Hubbard and van der Waals corrections vs hybrid functionals. J. Chem. Theory Comput. 12, 3807-3812 (2016).

57. Wei, C. \& Xu, Z. J. The comprehensive understanding of as an evaluation parameter for electrochemical water splitting. Small Methods 2, 1800168 (2018).

58. Bode, H., Dehmelt, K. \& Witte, J. Zur kenntnis der nickelhydroxidelektrodeI.Über das nickel (II)-hydroxidhydrat. Electrochim. Acta 11, 1079-1087 (1966).

59. Oskar, G. \& Josef, E. Die Struktur höherer Nickelhydroxyde. Z. f.ür. anorganische Chem. 261, 43-51 (1950).

60. Delaplane, R. G., Ibers, J. A., Ferraro, J. R. \& Rush, J. J. Diffraction and spectroscopic studies of the cobaltic acid system $\mathrm{HCoC}_{2}-\mathrm{DCoO}_{2}$. J. Chem Phys. 50, 1920-1927 (1969).

61. Wang, D. et al. In situ X-ray absorption near-edge structure study of advanced $\mathrm{NiFe}(\mathrm{OH}) \mathrm{x}$ electrocatalyst on carbon paper for water oxidation. J. Phys. Chem. C. 119, 19573-19583 (2015).

62. Gonzalez-Flores, D. et al. Nickel-iron catalysts for electrochemical water oxidation - redox synergism investigated by in situ X-ray spectroscopy with millisecond time resolution. Sustain. Energ. Fuels 2, 1986-1994 (2018).

63. Chen, J. Y. C. et al. Operando analysis of NiFe and Fe oxyhydroxide electrocatalysts for water oxidation: detection of Fe4+ by Mössbauer spectroscopy. J. Am. Chem. Soc. 137, 15090-15093 (2015). 
64. Louie, M. W. \& Bell, A. T. An investigation of thin-film Ni-Fe oxide catalysts for the electrochemical evolution of oxygen. J. Am. Chem. Soc. 135 , 12329-12337 (2013).

65. Risch, M. et al. Water oxidation by amorphous cobalt-based oxides: in situ tracking of redox transitions and mode of catalysis. Energy Environ. Sci. 8, 661-674 (2015).

66. Corrigan, D. A. The catalysis of the oxygen evolution reaction by iron impurities in thin film nickel oxide electrodes. J. Electrochem. Soc. 134, 377-384 (1987).

67. Berry, J. F. et al. An octahedral coordination complex of iron(VI). Science 312, 1937-1941 (2006).

68. Riedel, S. \& Kaupp, M. The highest oxidation states of the transition metal elements. Coord. Chem. Rev. 253, 606-624 (2009).

69. Hunter, B. M. et al. Trapping an iron(VI) water-splitting intermediate in nonaqueous media. Joule 2, 747-763 (2018).

70. Van der Ven, A., Morgan, D., Meng, Y. S. \& Ceder, G. Phase stability of nickel hydroxides and oxyhydroxides. J. Electrochem. Soc. 153, A210-A215 (2006).

71. Wang, H. et al. Transition-metal doped edge sites in vertically aligned MoS2 catalysts for enhanced hydrogen evolution. Nano Res. 8, 566-575 (2015).

72. Man, I. C. et al. Universality in oxygen evolution electrocatalysis on oxide surfaces. ChemCatChem 3, 1159-1165 (2011).

73. Dionigi, F., Reier, T., Pawolek, Z., Gliech, M. \& Strasser, P. Design criteria, operating conditions, and nickel-iron hydroxide catalyst materials for selective seawater electrolysis. Chemsuschem 9, 962-972 (2016).

74. Ma, R. et al. Topochemical synthesis of monometallic (Co2+-Co3+) layered double hydroxide and its exfoliation into positively charged $\mathrm{Co}(\mathrm{OH}) 2$ nanosheets. Angew. Chem. Int. Ed. Engl. 47, 86-89 (2008).

75. Trotochaud, L., Young, S. L., Ranney, J. K. \& Boettcher, S. W. Nickel-iron oxyhydroxide oxygen-evolution electrocatalysts: the role of intentional and incidental iron incorporation. J. Am. Chem. Soc. 136, 6744-6753 (2014).

76. Burke, M. S., Kast, M. G., Trotochaud, L., Smith, A. M. \& Boettcher, S. W. Cobalt-iron (oxy)hydroxide oxygen evolution electrocatalysts: the role of structure and composition on activity, stability, and mechanism. J. Am. Chem. Soc. 137, 3638-3648 (2015).

77. Bergmann, A. et al. Reversible amorphization and the catalytically active state of crystalline Co3O4 during oxygen evolution. Nat Commun 6, 8625 (2015).

78. Klimes, J., Bowler, D. R. \& Michaelides, A. Van der Waals density functionals applied to solids. Phys. Rev. B 83, 195131 (2011).

\section{Acknowledgements}

The WAXS experiments were performed on beamline ID31 at the European Synchrotron Radiation Facility (ESRF), Grenoble, France. We thank ESRF and HZB Bessy II for allocation of synchrotron radiation beamtime, and E. Hornberger and H. Schmies for their help during beamtimes. We also thank Dr. M. Görlin for the scientific and helpful discussions on NiFe (oxy)hydroxides. ZELMI of Technical University Berlin is acknowledged for their support with TEM measurements. Help at the beamline from Lukas Pielsticker (RUB) is appreciated, as well as the technical support from Dr Carlo Marini and Dr Nitya Ramanan at CLAESS beamline of ALBA synchrotron during the operando XAS measurements. The operando XAS work has funded by the European Research Council under grant ERC-OPERANDOCAT (ERC-725915). S.K. acknowledges funding from the IMPRS SurMat. This work was partially supported by the German Research Foundation (DFG, Deutsche Forschungsgemeinschaf) in the frame of the collaborative research center/transregio TRR247 Heterogeneous Oxidation Catalysis in the liquid Phase, project no. 388390466 and through grant reference number STR 596/8-1, Bifunctional seawater electrolyzer, STR 596/12-1, catalyst-support interactions on the activity and stability of water splitting catalysts, and by the Federal Ministry for economic affairs and energy (Bundesministerium für Wirtschaft und Energie, BMWi) under grant number 03EIV041F, MethFuel/ MethQuest. Work at Purdue was supported through the Office of Science, Office of Basic Energy Sciences, Chemical, Biological, and Geosciences Division under DE-SC0010379 (J.G.). Work at University of Science and Technology of China was supported by the National Key R\&D Program of China (2018YFA0208603) and the Frontier Science Key Project of the Chinese Academy of Sciences (QYZDJSSW-SLH054). F.D. and P.S. acknowledge partial funding by the Deutsche Forschungsgemeinschaft (DFG, German Research Foundation) under Germany's Excellence Strategy - EXC 2008/1 -390540038.

\section{Author contributions}

F.D. conceived and performed the operando WAXS measurements at ESRF and the analysis of the operando WAXS data including Rietveld refinement. Z.Z. conceived DFT calculations. Z.Z. and D.F. performed DFT calculations of structure search. Z.Z., S.D. and J.Z. performed the DFT calculations of OER mechanism. I.S. performed the operando XAS experiments at ALBA, analyzed the corresponding data, and wrote part of the manuscript. T.M. and H.S. synthesized all the samples and performed the RDE electrochemical characterization. M.B.L., S.K. and I.Z. performed the operando XAS experiments at ALBA. T.M., A.B. and J.D. performed the operando WAXS measurements at ESRF. J.F.d.A. designed and performed the DEMS experiments. M.G. performed the TEM. D.T. designed and performed the sXAS measurements at BESSY II and wrote part of the manuscript. F.D., B.R.C., and P.S. designed the research and experiments and wrote parts of the manuscript. Z.Z., W.-X.L. and J.G. designed the research and DFT calculations, and wrote part of the manuscript. All authors discussed the results and assisted during manuscript preparation.

\section{Competing interests}

The authors declare no competing interests.

\section{Additional information}

Supplementary information is available for this paper at https://doi.org/10.1038/s41467020-16237-1.

Correspondence and requests for materials should be addressed to F.D., Z.Z., B.R.C. or P.S.

Peer review information Nautre Communications thanks the anonymous reviewers for their contributions to the peer review of this work.

Reprints and permission information is available at http://www.nature.com/reprints

Publisher's note Springer Nature remains neutral with regard to jurisdictional claims in published maps and institutional affiliations.

Open Access This article is licensed under a Creative Commons Attribution 4.0 International License, which permits use, sharing, adaptation, distribution and reproduction in any medium or format, as long as you give appropriate credit to the original author(s) and the source, provide a link to the Creative Commons license, and indicate if changes were made. The images or other third party material in this article are included in the article's Creative Commons license, unless indicated otherwise in a credit line to the material. If material is not included in the article's Creative Commons license and your intended use is not permitted by statutory regulation or exceeds the permitted use, you will need to obtain permission directly from the copyright holder. To view a copy of this license, visit http://creativecommons.org/ licenses/by/4.0/.

(C) The Author(s) 2020 